\title{
A Comprehensive Error Compensation System for Correcting Geometric, Thermal, and Cutting Force-Induced Errors
}

\author{
J. C. Liang, H. F. Li, J. X. Yuan and J. Ni \\ Department of Mechanical Engineering and Applied Mechanics, The University of Michigan, Ann Arbor, USA
}

\begin{abstract}
A comprehensive error compensation system has been developed to correct geometric, thermal, and cutting forceinduced errors on a turning centre. The basic approach to error compensation is proposed in this paper. The implementation of error compensation control and of hardware configuration of the system are also presented. A total of 11 geometric and thermal error components and 10 cutting forceinduced error components can be compensated for using this system. Performance evaluations have been carried out using actual cutting tests. Experimental results show that the diameter accuracy of the part has been improved more than 5 times and taper accuracy of the part has been improved about 5 times.
\end{abstract}

Keywords: Cutting force-induced error; Error compensation; Geometric error; Thermal error; Turning centre

\section{Introduction}

Among the many sources of machine tool errors, thermal deformation and geometric errors are traditionally known as key contributors. It is reported that thermal errors could contribute $40-70 \%$ of workpiece errors in precision machining. Geometric errors are caused by the inaccuracy of machine parts, misalignment of parts, and improper assembly of the machine. The ballscrew lead error compensation and reversal error compensation by the $\mathrm{CNC}$ controller can reduce some of the geometric errors, however, the squareness and parallelism errors, slide angular errors, and straightness errors still remain.

Real-time error compensation (RTEC) techniques which can correct volumetric or planar errors offer a more effective way of enhancing machine accuracy and have received wide attention in recent years complementing the machine design and manufacturing approach. RTEC techniques for geometric and thermal (G-T) errors have successfully improved machine tool accuracy by up to one order of magnitude [1-3].

Correspondence and offprint requests to: Dr J. C. Liang, 2041 G. G. Brown Building, University of Michigan, Ann Arbor, MI 481092125 , USA.
Besides G-T errors, cutting force-induced (F) error is another major source of machine tool error. However, most of the current error compensation research has been focused on G.T error compensation, with very little work on cutting forceinduced error compensation [4]. Additionally, there was no complete compensation system for correcting $\mathrm{G}-\mathrm{T}$ and $\mathrm{F}$ errors. This was mainly due to the following:

1. In finish machining, the cutting force is small and the resulting deflection can be neglected.

2. The cutting force-induced error is more or less repeatable for the same batch of parts.

3. Initial error compensation research was complicated by including cutting force-induced error.

However, the above reasons are no longer valid in today"s manufacturing and research environment. First of all, hard turning is gaining popularity in many of today's finishing operations. It is commonly used to cut hardened steel directly to its final form and finish, thus avoiding the customary grinding operations. The cutting forces in hard turning operations can be very high and thus cannot be neglected. Secondly, the cutting force-induced errors are no longer repeatable in flexible manufacturing systems where a variety of parts may be machined on the same machine. Finally, combining G-T error compensation with $F$ error compensation is no longer complicated, based on many years of research.

In this paper, a comprehensive advanced error compensation system that can compensate for G-T and F errors in real-time is developed on a turning centre. The basic approach of the comprehensive error compensation will be presented in section 2. The implementation of the error compensation system will be introduced in section 3. The effectiveness of the proposed error compensation system will be demonstrated in section 4 . Some conclusions will be presented in section 5 .

\section{Basic Approach of Comprehensive Error Compensation}

The basic approach of the comprehensive error compensation method is shown in Fig. 1. It can be explained as follows: 


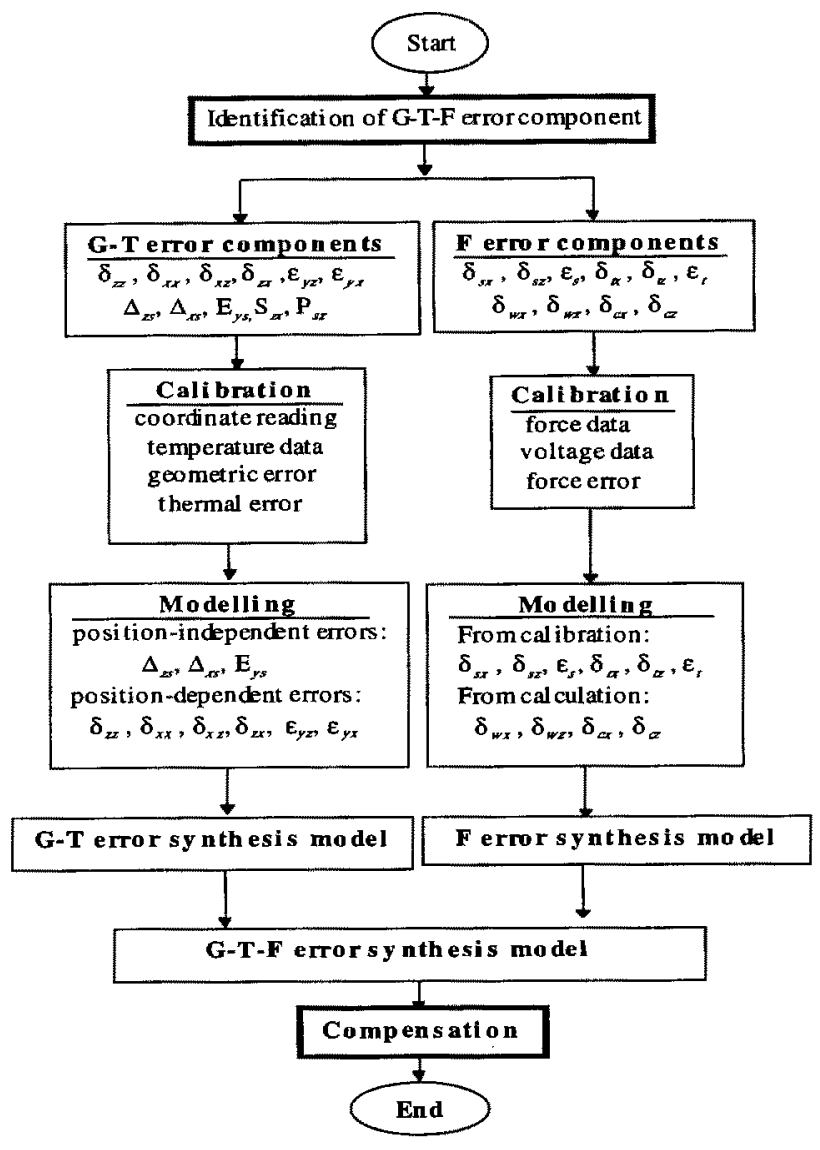

Fig. 1. Basic approach of G-T-F error compensation.

1. The first step is identification of the G-T-F error components. There are a total of 11 G-T error components identified for this turning centre. They are:

Linear displacement error $\delta_{z z}, \delta_{x x}$

Straightness error $\delta_{x z}, \delta_{z x}$

Yaw error $\varepsilon_{y z}, \varepsilon_{y x}$

Squareness error between $Z$ - and $X$-axes $S_{z x}$

Parallelism error between $Z$ - and spindle-axes $P_{s z}$

Spindle and origin drift errors in Z-direction $\Delta_{z s}$

Spindle and origin drift errors in $X$-direction $\Delta_{x s}$

Spindle inclination error $E_{y s}$

There are a total of 10 cutting force-induced error components identified in this research. They are:

Spindle deflection errors $\delta_{s x}, \delta_{s z}$

Spindle rotational error $\boldsymbol{\varepsilon}_{s}$

Tool post deflection errors $\delta_{t x}, \delta_{t z}$

Tool post rotational error $\varepsilon_{t}$

Workpiece deflection errors $\delta_{w x}, \delta_{w z}$

Cutting tool deflection errors $\delta_{c x}, \delta_{c z}$

2. The next step in error compensation is the calibration of G-T-F error components. Two sets of data must be obtained in this step. One is the data of the geometric, thermal, and

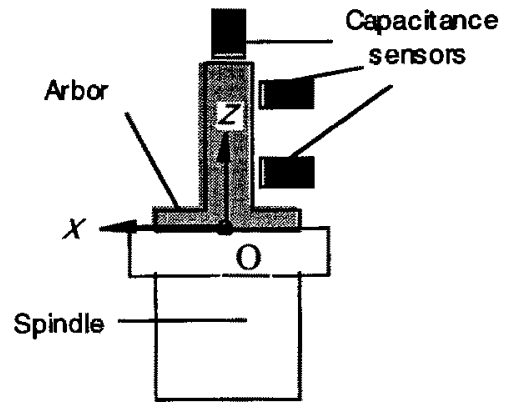

Fig. 2. Set-up for spindle and origin drift error calibration.

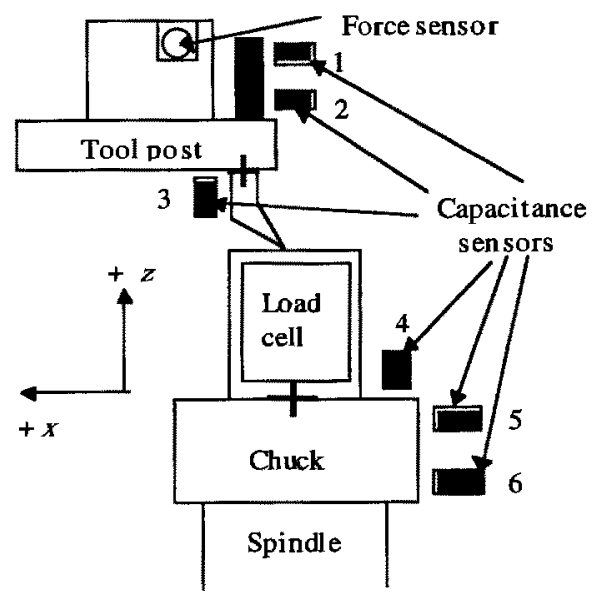

Fig. 3. Set-up for cutting force-induced error calibration.

cutting force-induced errors. The other is the data related to the G-T-F errors, such as temperature data, cutting force data, coordinate readings, and so on.

Some sensors and instruments such as thermistors, capacitance sensors, load cells, and laser measurement systems are used here to obtain these data. Figure 2 shows the set-up for spindle and origin drift error calibration which is used to pick up spindle and origin drift error data $\Delta_{z s}, \Delta_{x s}$, and $E_{y s}$. The other G-T error components are calibrated by a laser measurement system. Figure 3 shows the set-up for cutting forceinduced error calibration which is used to obtain cutting forceinduced errors $\delta_{s x}, \delta_{s z}, \varepsilon_{s}, \delta_{t x}, \delta_{t z}$, and $\varepsilon_{t}$ of the spindle and tool post. The other cutting force-induced error components are obtained by calculation using the cantilever beam assumption.

3. The third step of error compensation is modelling for the G-T-F error components. After the data is obtained, the error component models are fitted based on the data.

There are $11 \mathrm{G}-\mathrm{T}$ error components for this turning centre, each error component should have its own model except for squareness error and parallelism error.

Usually position-independent thermal error components can be modelled as

$$
\delta=a_{0}+\sum_{i=1}^{n} b_{i} \Delta T_{i}+\sum_{i=1}^{n} \sum_{j=1}^{n} c_{i j} \Delta T_{i} \Delta T_{j}+\ldots
$$

where

$\delta=$ thermal error, 
$a_{0}, b_{i}, c_{i j}=$ coefficients of the model,

$\Delta T_{i}, \Delta T_{j}=$ temperature rise at some key points on the machine

The position-dependent thermal error components can be modelled as

$$
\begin{aligned}
\delta & =\delta_{G}+\delta_{T} \\
\delta_{G} & =a_{0}+a_{1} x+a_{2} x^{2}+\ldots \\
\Delta_{T} & =K_{T} x \\
K_{T} & =b_{0}+\sum_{i=1}^{n} c_{i} \Delta T_{i}+\sum_{i=1}^{n} \sum_{j=1}^{n} d_{i j} \Delta T_{i} \Delta T_{j}+\ldots
\end{aligned}
$$

where

$\delta, \delta_{G}, \delta_{T}=$ total error, geometric error, and thermal error $x, a_{0}, a_{1}, \ldots=$ slide position and coefficients of the model $K_{T}=$ variant slope of the error curve due to thermal error $\Delta T_{i}, \Delta T_{j}=$ temperature rise at some key points on the machine $b_{0}, c_{i}, d_{i j}=$ coefficients of the model of the time-variant slope

For the cutting force-induced error components, two multiple regression matrix models of order 3 are fitted for the error components on the spindle and tool post, respectively. The general form of the model is as follows:

$$
\left\{\begin{array}{l}
\delta_{x} \\
\delta_{z} \\
\varepsilon
\end{array}\right\}=\left[\begin{array}{lll}
c_{11} & c_{12} & c_{13} \\
c_{21} & c_{22} & c_{23} \\
c_{31} & c_{32} & c_{33}
\end{array}\right]\left\{\begin{array}{c}
F_{x} \\
F_{z} \\
M_{y}
\end{array}\right\}
$$

where

$$
\begin{aligned}
& \delta_{x}, \delta_{z}=\text { deflection errors in } x \text { - and } z \text {-direction } \\
& \varepsilon=\text { rotational errors } \\
& c_{i j}=\text { coefficients of the regression model } \\
& F_{x}, F_{z}=\text { cutting forces in } x \text { - and } z \text {-direction, } \\
& M_{y}=\text { moment around the } y \text {-direction }
\end{aligned}
$$

For real-time error compensation, the error components should be combined using error synthesis models. This can usually be obtained using the kinematics rigid-body assumption. The G-T error synthesis model and F error synthesis model should be obtained, respectively.

The G-T error synthesis models are:

$$
\begin{aligned}
\Delta W_{x}^{G T}=\delta_{x z}+\delta_{x x}+P_{s z} z & +\left(\varepsilon_{y z}+\varepsilon_{y x}-E_{y s}\right) T_{z}+\Delta_{x s} \\
& +\left(\varepsilon_{y z}-E_{y s}\right) M_{c t z}-M_{r c z} E_{y s} \\
\Delta W_{z}^{G T}=\delta_{z x} & +\delta_{z z}-\left(S_{z x}+\varepsilon_{y z}\right) x-\left(\varepsilon_{y z}+\varepsilon_{y x}-E_{y s}\right) T_{x} \\
& +\Delta_{z s}-\left(\varepsilon_{y z}-E_{y s}\right) M_{c t x}+M_{r c x} E_{y s}
\end{aligned}
$$

where

$T_{x}, T_{z}=$ coordinates of the tool tip in the tool turret coordinate system

$M_{c t x}, M_{c t z}=$ offsets of the tool turret origin in the $X$ - and $Z$ directions in the carriage system

$M_{r c x}, M_{r c z}=$ offsets of the carriage origin in the $X$ - and $Z$ directions in the reference coordinate system

The F error synthesis models are:

$$
\begin{aligned}
& \Delta W_{x}^{F}=\delta_{c x}+\delta_{t x}-\delta_{w x}-\delta_{s x}+\varepsilon_{s} T_{z}+\varepsilon_{t} T_{z} \\
& \Delta W_{z}^{F}=\delta_{c z}+\delta_{t z}-\delta_{w z}-\delta_{s z}+\varepsilon_{s} T_{x}+\varepsilon_{t} T_{x}
\end{aligned}
$$

where $T_{x}$ and $T_{z}$ are the same as above.

All the error components in G-T and F error synthesis models are described in step 1.

By combining them, the G-T-F error synthesis models can be obtained as follows:

$$
\begin{aligned}
& \Delta W_{X}=\Delta W_{X}^{G T}+\Delta W_{X}^{F} \\
& \Delta W_{Z}=\Delta W_{Z}^{G T}+\Delta W_{Z}^{F}
\end{aligned}
$$

4. The fourth step is compensation control. It can be done in real-time when all the models are obtained. The computer collects the temperature and cutting force data from the sensors on the machine and the coordinate readings $x$ and $z$ from the controller. After calculation using models, the compensation signals $\Delta X$ and $\Delta Z$ are sent to the $C N C$ controller to correct the G-T-F errors.

\section{Implementation of Error Compensation System}

The comprehensive G-T-F error compensation system has been made by integrating an IBM/PC computer with the existing $\mathrm{CNC}$ controller on the turning centre. This includes sensing, modelling, calibration, and control systems. The hardware configuration of the system is shown in Fig. 4. This comprehensive error compensation system can perform the following functions:

Collects the temperature and cutting force information from the thermistors and the piezoelectric force sensor in real-time. Reads the current position of the slides from the controller. Obtains the error data from capacitance sensor and laser measurement system for calibration purposes.

Calculates all the G-T-F error components using mathematical models.

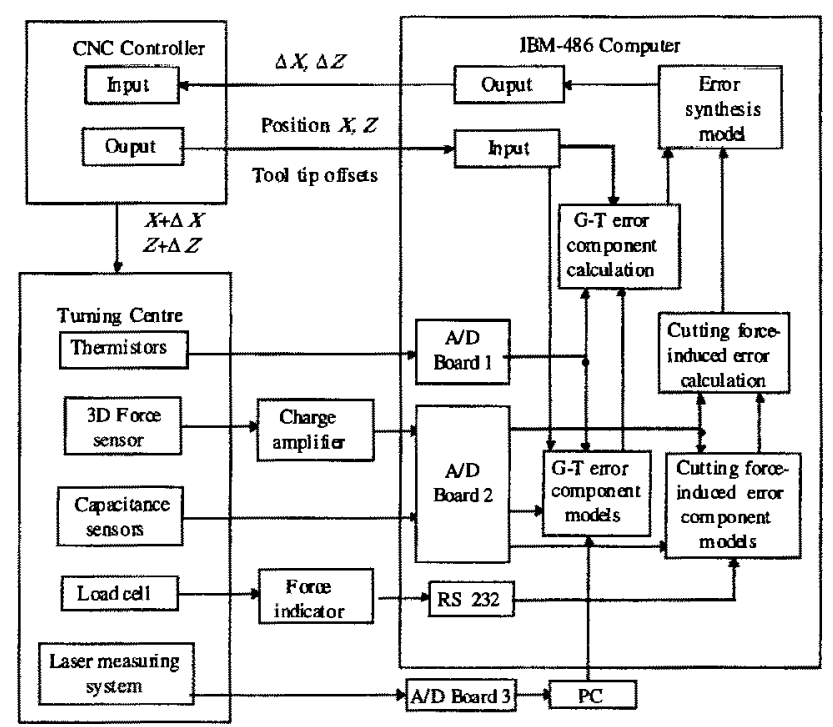

Fig. 4. The hardware configuration of the error compensation system. 
Obtains the planar errors in two directions using error synthesis models.

Transfers the planar error signals to the CNC controller to implement the planar error compensation in real-time.

During machining, the machine runs according to the part program stored in the $\mathrm{CNC}$ controller. The parameters of the G-T-F error component mathematical models, which are precalibrated off-line by using the calibration system, are stored in the database of the IBM/PC computer. The temperature and the cutting force of the turning centre is measured on-line by the thermistor and the piezoelectric force sensor, and the data is sent to the computer through two $\mathrm{A} / \mathrm{D}$ boards. The positions of the slides are obtained directly from the controller. Based on measurements of the temperatures and the cutting forces, slide positions, and the mathematical models, all G-T-F error components can be calculated. The amount of the total G-T-F errors between the tool tip and the workpiece in two directions can then be determined using the G-T-F planar error synthesis model. The resulting compensation signals for the two axes are sent to the programmable logic controller (PLC) through a digital input/output interface. Once the PLC receives the compensated error values, the nominal positions are corrected by the PLC program and transferred to the computer numerical controller (CNC). The CNC controller reads the compensation signal and shifts the origins of the $X-, Z$-axes to implement the compensation.

\section{Performance Evaluation of the Error Compensation System}

Several types of test have been done in order to evaluate the effectiveness of the error compensation system in compensating for G-T and $\mathrm{F}$ errors. The experimental results will be explained as follows.

\subsection{G-T Error Compensation Test}

A G-T error compensation test has been done on the turning centre. The cutting conditions are: spindle speed $n=1200$ r.p.m., depth of cut $d=0.005$ inch, and feedrate $f=10 \mathrm{inch} / \mathrm{min}$. The test began in a cold condition and ran to a warm condition. The parts are cut every half hour without and with compensation, respectively. In total, the test took $5 \frac{1}{2} \mathrm{~h}$. The experimental results are shown in Fig. 5. It can be seen that without compensation the diameter of the part becomes larger and larger as time increases, but with compensation the diameter of the part changes only a little. The diameter error range of the part is $1.0 \times 10^{-3}$ in. without error compensation; while it is $0.2 \times 10^{-3}$ in. with error compensation.

\subsection{Cutting Force-Induced Error Compensation Test}

A cutting force-induced error compensation performance test has also been done on the turning centre. In order to verify the effectiveness of the compensation system, three types of

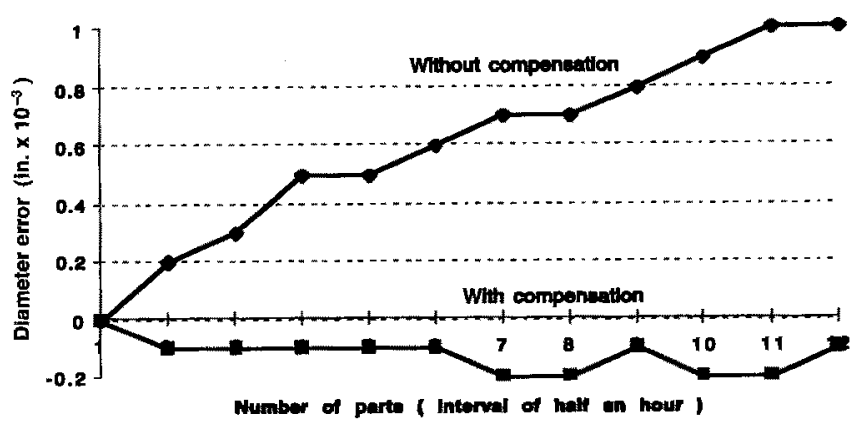

Fig. 5. Performance of G-T error compensation.

test part are designed. The shape of the first type of part is a cylinder, which means that the depth of cut is constant. The shape of the second type of part is a taper, which means the depth of cut changes continuously when the part is cut in the vertical direction $(0.01-0.06 \mathrm{in})$. The shape of the third type of part is stepped, which means that the depth of cut changes when the part is cut at different steps $(0.02,0.04,0.06$ in.). The cutting conditions and the experimental results are shown in Table 1. Since this type of performance test has been done in a short time, the G-T errors were taken off.

From Table 1 it can be seen that the maximum taper of the part is $1.3 \times 10^{-3}$ in. without compensation and reduced to $0.2 \times 10^{-3}$ in. with cutting force-error compensation.

\subsection{G-T and Cutting Force-Induced Error Compensation Test}

After the separated G-T error compensation and cutting forceinduced error compensation tests, the combined G-T-F error compensation test is done on the turning centre, which means both the G-T error and F error are compensated for at the same time. The cutting conditions are: spindle speed $n=1200$ r.p.m., feedrate $f=10 \mathrm{in} . / \mathrm{min}$, and depth of cut $d=0.04 \mathrm{in}$. The test began in a cold condition and ran to a warm condition. The parts are cut every $50 \mathrm{~min}$. In total, the test took more than

Table 1. Experimental results of cutting force-induced error compensation (unit: inch).

\begin{tabular}{|c|c|c|c|c|c|}
\hline \multirow[t]{2}{*}{ Type } & \multirow[t]{2}{*}{ W/O } & \multicolumn{3}{|c|}{ W } & \multirow[t]{2}{*}{ Cutting parameter } \\
\hline & & No. 1 & No. 2 & No. 3 & \\
\hline & 1.5500 & 1.5502 & 1.5502 & 1.5501 & $n=750$ r.p.m. \\
\hline & 1.5491 & 1.5501 & 1.5501 & 1.5500 & $f=7.5 \mathrm{in} / \mathrm{min}$ \\
\hline & 1.5487 & 1.5503 & 1.5502 & 1.5499 & $d=0.05$ \\
\hline & 1.5279 & 1.5284 & 1.5285 & 1.5283 & $n=750$ r.p.m. \\
\hline & 1.5276 & 1.5285 & 1.5286 & 1.5285 & $f=5 \mathrm{in} / \mathrm{min}$ \\
\hline & 1.5270 & 1.5285 & 1.5287 & 1.5285 & $d=0.01-0.06$ \\
\hline & 1.5481 & 1.5482 & 1.5484 & 1.5482 & $n=750$ r.p.m. \\
\hline & 1.5480 & 1.5484 & 1.5484 & 1.5484 & $f=4 \mathrm{in} / \mathrm{min}$ \\
\hline & 1.5470 & 1.5486 & 1.5483 & 1.5481 & $d=0.02-0.06$ \\
\hline
\end{tabular}




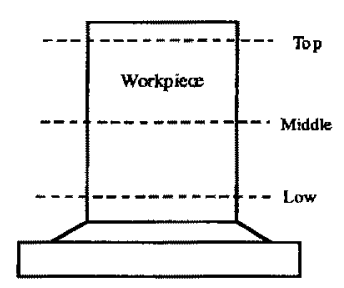

Fig. 6. Experimental workpiece.

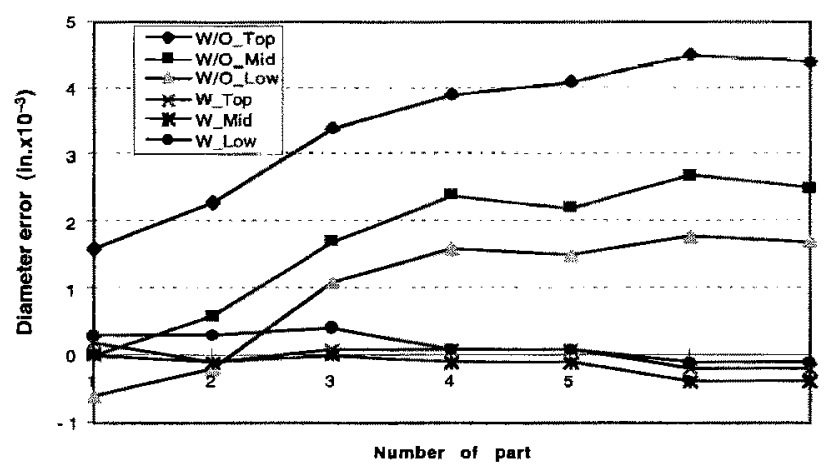

Fig. 7. Performance of G-T and cutting force-induced error compensation.

$5 \mathrm{~h}$. The data of experimental results are obtained from three sections for each part: top section, middle section, and low section which are shown as in Fig. 6. The experimental results are shown in Fig. 7 .

From Fig. 7, it can be seen that without compensation the diameter error of the part becomes larger and larger as time increases owing to thermal drift error, but with compensation the diameter error of the part changes only a little.

The taper error of the part without compensation is much larger than that with compensation owing to cutting forceinduced error and also thermal tilt error. Overall, the diameter error range of the part without compensation is $2.9 \times 10^{-3}$ in. and the maximum taper is $2.8 \times 10^{-3}$ in. The diameter error range of the part with compensation is $0.5 \times 10^{-3} \mathrm{in}$. It means we can get good results by using the G-T and F error compensation no matter if the error components are G$\mathrm{T}$ errors, $\mathrm{F}$ error, or both of them.

\section{Conclusion}

A comprehensive error compensation system has been developed for the first time to compensate for G-T-F errors on a turning centre. A basic approach to error compensation has been proposed that can be used for different $\mathrm{CNC}$ machine tools. Several types of tests have been done. It can be seen from the experimental results that with the combined G-T and $F$ error compensation, the diameter accuracy of the part has been improved more than 5 times and taper accuracy improved about 5 times. Also, the cutting force-induced error is not negligible in some conditions. Currently, this comprehensive error commpensation system is being commercialized in a machine tool plant.

\section{Rèfèrences}

1. M. A. Donmez, D. S. Blomquist, R. J. Hocken, C. R. Liu and M. M. Barash, "A general methodology for machine tool accuracy enhancement by error compensation", Precision Engineering, 8(4) pp. 187-196, 1986.

2. J. S. Chen, J. Yuan, I. Ni and S. M. Wu, "Real-time compensation for time-variant volumetric error on a machining center", ASME Journal of Engineering for Industry, 114, pp. 472-479, 1993.

3. C. H. Lo, J. Yuan and J. Ni, "An application of real-time error compensation on a turning center", International Journal of Machine Tools Manufacturing, 35(12), pp. 1669-1682, 1995.

4. S. Yang, J. Yuan and J. Ni, "Real-time cutting force-induced error compensation on a turning center", Proceedings of Second S. $M$. Wu Symposium on Manufacturing Science, vol. 2, pp. 50-56, 1996. 